\title{
«Pena y gloria trocadas»: la ciudad en El condenado por desconfiado de Tirso de Molina
}

\section{«Pain and Glory Exchanged»: The City in The Condamned by Distrustful by Tirso de Molina}

\section{Isabelle Bouchiba-Fochesato}

Université Bordeaux Montaigne EA 3656 AMERIBER (GRIAL)

FRANCIA

Isabelle.Bouchiba-Fochesato@u-bordeaux-montaigne.fr

[Hipogrifo, (issn: 2328-1308), 8.2, 2020, pp. 39-51]

Recibido: 19-12-2019 / Aceptado: 27-01-2020

DOI: http://dx.doi.org/10.13035/H.2020.08.02.04

Resumen. En este artículo se intenta mostrar que en la obra tirsiana El condenado por desconfiado se produce un intercambio de las cualidades poético-religiosas tradicionalmente asociadas con el monte (cuna de la santidad eremítica) y con la urbe (sede de todos los vicios y pecados). Sin embargo, mostramos también que la obra tampoco se limita a una mera inversión de las polaridades entre los dos espacios, sino que hace, más bien, una nueva propuesta en la que, el monte va desapareciendo como símbolo operativo de la santidad, mientras que la ciudad se va imponiendo como posible espacio de redención en una modernidad temible.

Palabras clave. Monte; ciudad; Condenado por desconfiado; modernidad.

Abstract. This article attempts to show that in the Tirsian play, El condenado por desconfiado, there is an exchange of the poetic-religious qualities traditionally associated with the mountain (eremitic holiness) and with the city (seat of all vices and sins). However, we also show that the play is not limited to a mere inversion of the polarities between the two spaces but rather makes a new proposal in which, the mountain disappears as an operational symbol of holiness, while the city is imposed as a possible redemption space in a fearsome modernity.

Keywords. Mount; City; Condenado por desconfiado; Modernity. 
La ciudad en la literatura aurisecular, ya se trate de novelas, comedias o ensayos - pero aquí se tratará de teatro-, es un escenario recurrente, por no decir omnipresente. La ciudad es un elemento cultural complejo. Bien se sabe que el pensamiento renacentista suele identificarla con el epicentro de la hipocresía, de la violencia física y simbólica y de la lujuria mientras que el campo, "la aldea", aúna, en perfecta simetría, todas las virtudes opuestas de honestidad, esfuerzo y humildad. Basta con recordar la obra famosísima del cantábrico fray Antonio de Guevara Menosprecio de corte y alabanza de aldea, cuyo título lo resume todo. Esta construcción ideológica, la convoca el mismo Cervantes cuando, en el Quijote, hace que el cura exclame: «ya yo sé de experiencia que los montes crían letrados y las cabañas de los pastores encierran filósofos» $(I, 50)$. El hecho de que el que emite esta verdad sea uno de los personajes indudablemente sano y serio de la obra no deja lugar a dudas sobre el nivel de credibilidad de esta declaración. Es innegable que, en sociedades de pensamiento judeocristiano, la ciudad hereda características de su pasado bíblico. Huelga recordar que es Caín, después de asesinar a su hermano Abel y de ser condenado a vagabundear por la tierra por Dios, quien funda la primera ciudad, en el Este del Edén, Enoc. Aunque la rama de Caín desaparece con el diluvio, las ciudades de Sodoma y Gomorra bastan para fijar una percepción eminentemente problemática de la urbe y de sus habitantes. No hay ciudades en el Jardín del Edén y Cristo es el buen pastor, no es un habitante de la ciudad. En la comedia nueva, las ciudades suelen ser el escenario de comedias de enredo desencadenadas en las que, muy a menudo, el gracioso, gran conocedor de los vicios de la sociedad, no deja de informar a su amo, con mucha comicidad, de los peligros de la vida urbana (particularmente cuando se trata de Madrid) -como en las primeras escenas de La celosa de sí misma' - o de su inmoralidad (a menudo cuando se trata de Toledo) como por ejemplo en La villana de la Sagra. Nuestro propósito no consistirá en desarrollar esta temática muy presente en las comedias tirsianas sino analizar el movimiento opuesto que se manifiesta, según intentaremos demostrar, en uno de sus dos grandes dramas teológicos, El condenado por desconfiado. Bien se recuerda que esta obra pone en escena a dos protagonistas totalmente opuestos, el ermitaño Paulo y el rufián Enrico, inscritos cada uno en un biotopo tradicionalmente asociado con su actividad dramática: el monte para aquél, la ciudad para éste. Recordemos primero brevemente el argumento de la obra. Algún día, tras diez años de vida perfectamente ascética en el monte, Paulo se atreve a pedirle a Dios la confirmación de su salvación. El demonio aprovecha la ocasión para aparecerle bajo la forma de un ángel y anunciarle al ermitaño que su destino será exactamente el mismo que el de un tal Enrico, hijo de Anareto, que vive en la ciudad de Nápoles. Una vez en Nápoles, Paulo descubre que Enrico resulta ser uno de los peores rufianes de la ciudad. Desesperado por lo que considera ser una promesa de damnación, Paulo vuelve a su monte y se convierte en bandido convencido de actuar en consonancia con la seudo-promesa divina. Al final de la obra, en un movimiento de inversión dramatizado en tres actos, Paulo inicialmente 
prometido a la santidad acaba condenado (nunca supo arrepentirse) mientras que el asesino Enrico muere en estado de gracia por su amor sincero a su padre.

Si el gran hispanista Menéndez Pidal, en su discurso de entrada en la Real Academia ${ }^{2}$, analiza muy bien la tradición folklórica multisecular en la que se inscribe esta historia, raras veces se interesan los especialistas en los espacios antagónicos en los que se desarrolla. Es precisamente lo que nos proponemos hacer en esta ponencia en la que vamos a intentar estudiar cómo la ciudad, esta contraposición del monte eremítico, se va convirtiendo a lo largo de las tres jornadas de la obra en un espacio mucho más híbrido de lo que parece. Para ello, veremos en un primer momento cómo la ciudad, por oposición al monte, se ve primero asociada por el texto dramático con los atributos tradicionales evocados en esta introducción; luego veremos cómo se produce un movimiento de cambio de polarización de los dos espacios que sirven de marco principal en la obra; y por fin, en una tercera parte conclusiva intentaremos mostrar que quizás, en este drama, se trate para el dramaturgo, a través de una redistribución de los espacios simbólicos, de proponer una nueva percepción de la modernidad a través de un espacio clave, la urbe, y de los retos de esta modernidad a la vez temida y percibida como ineluctable.

\section{NÁPOLES, LA CAPITAL DEL CRIMEN}

La obra desarrolla su intriga en tres tipos de espacios principales: el monte, la ciudad de Nápoles y el mar (tanto sus orillas como el mismo espacio acuático). En Nápoles, cinco lugares constituyen el escenario de la acción: el patio de la casa de Celia, dama de Enrico, el rufián, cortesana - para no decir ramera-, la Puerta del Mar, la habitación de la casa del viejo Anareto, padre de Enrico, la calle y la cárcel (una celda primera, luego un calabozo). Tenemos ya, con esta mera evocación de los espacios urbanos la trayectoria plausible del personaje del bandido Enrico en su biotopo de predilección, la urbe. Ya no se trata de cualquier urbe, ya que se trata de Nápoles, ciudad marco del primer crimen de don Juan al principio de El burlador de Sevilla también atribuido a Tirso de Molina. La primera aparición de la ciudad en la obra se produce rápidamente después del inicio. El texto dramático no nos ofrece, sin embargo, una escena urbana sino la convocación de la palabra en una escena situada en pleno monte: Paulo, acosado por la duda después de diez años pasados en el monte, le pide a Dios que le confirme su salvación. Frente a tanta desconfianza, el "Juez Supremo" (cito) le permite al Demonio tentar al ermitaño. El Demonio aparece entonces bajo la forma de un ángel y declara:

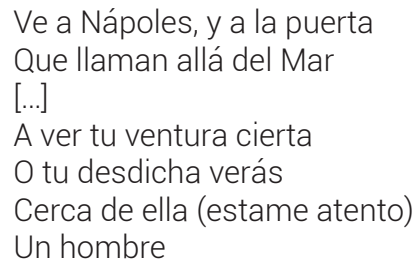




\section{[...] \\ Que Enrico tiene por nombre. \\ $[\ldots]$ \\ Dios, que en él repares, quiere \\ Porque el fin que aquél tuviere \\ Este fin has de tener ${ }^{3}$.}

La intervención inicial del Demonio que impone la ciudad de Nápoles como segundo marco principal de la acción, en la economía global de su engaño, participa claramente de la inscripción de la ciudad en el tópico del lugar tradicional del pecado. A partir de este momento el texto de la primera jornada nunca va a dejar de ofrecer al lector/espectador la percepción de esta urbe como biotopo indiscutible de los peores pecados y pecadores de este mundo dramático. Si la acción liminar (la presentación de Paulo, la verbalización de su angustia y la aparición del demonio) ocurría en el monte, lugar privilegiado de la soledad eremítica, se desplaza rápidamente a Nápoles, precisamente después de las últimas palabras del demonio, y nos encontramos de repente en el atrio de una casa. Esta casa, lo descubrimos rápidamente, es la de Celia, el único personaje femenino de la obra, así introducida por el texto:

La fama desta mujer

Solo a verla me ha traído 4 .

Quien pronuncia estos dos versos es el marqués Lisando acompañado de su amigo el marqués Octavio. Huelga subrayar la cadena de asociaciones implícitas que nos permiten adivinar de qué vive la "mujer" en cuestión: dos hombres, en un atrio, la palabra mujer en vez de dama y todo esto bajo el recuerdo subliminal de las últimas palabras del demonio, todos estos elementos bastan para eufemisar la actualización de un personaje de prostituta (tanto más que bien se sabe que la mujer -y aún más la mujer perversa- es el aliado tradicional del demonio). Se van a encadenar en el acto primero y en buena parte de los actos dos y tres todos los tópicos de la violencia y de la depravación urbanas. La casa de Celia primero, como acabamos de verlo, no es nada menos que un prostíbulo y es el sitio tanto de escenas de muy ambigua galantería por parte de los dos marqueses como de la violencia genérica - para convocar un concepto anacrónico pero eficaz- por parte de Enrico, amante de Celia que no vacila en tomarle a la dama todas las dádivas de sus admiradores. Hay que precisar para ser perfectamente honesta que Celia se los da de muy buen grado, de tan fascinada por la fuerza del rufián. Después de este primer lugar urbano, la acción se desplaza hacia la famosa Puerta del Mar que va a ser el marco de un clímax de violencia y de transgresión por parte de Enrico. Allí se encuentran para merendar Celia, Enrico y unos compañeros suyos, todos bandidos, ladrones y asesinos. La escena se inaugura entre gritos y espantos de los miembros de la compañía al ver a Enrico echar al mar -donde es indudable que se

3. Tirso de Molina, Obras dramáticas completas, t. III, p. 457.

4. Tirso de Molina, Obras dramáticas completas, t. III, p. 458. 
ahoga - a un pobre limosnero que se había atrevido a pedirle una limosna al rufián. Este crimen blasfematorio lo justifica así el protagonista:

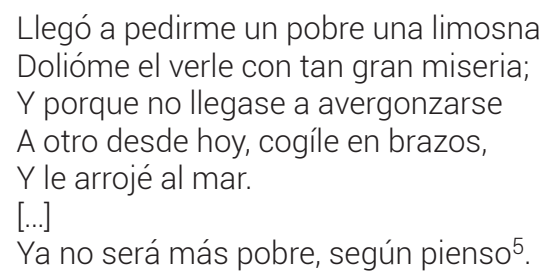

Claro está que esta violencia la exterioriza un protagonista de la obra, pero es cierto también que el lugar, la ciudad, constituye su condición de realización en la medida en que en ningún otro espacio dramático vamos a encontrar rufianes, mendigos, y como lo vamos a ver ahora, casas habitadas que incendiar, damas que violar o maridos que asesinar ${ }^{6}$. De estos crímenes abominables, entre otros, se jacta en efecto Enrico inmediatamente después del asesinato del mendigo en un concurso de recuerdo de atrocidades entre los rufianes del que resulta fácil vencedor.

La segunda jornada también participa de la inscripción de la ciudad de Nápoles en el marco tradicional de la ciudad peligrosa y viciada en particular mediante una escena callejera de asesinato por parte del espadachín. Antes de detenernos brevemente en esta escena, nos parece importante señalar que con los tres sitios aquí evocados, atrio de una casa, Puerta del Mar y calle, tenemos evocada, de manera metonímica, la ciudad entera, es decir a la vez el conjunto de sitios constitutivos de la vida en la sociedad urbana y a la vez todas las casas, potencialmente invadidas por el vicio, todos los lugares de ocio, excesivamente permisivos, y todas las calles, espacio peligroso por definición para quien se encuentra solo en él después de la puesta del sol. Esta geografía es aún más reveladora si pensamos en los lugares ausentes de esta construcción metonímica y en particular las iglesias. Sea lo que fuere, en la escena a la que nos estamos refiriendo, Enrique, pagado para matar a un hombre, detiene su brazo en el último momento por ser su víctima un hombre de la edad de su padre. Este acto de piedad no le impide sin embargo asesinar, acto seguido, al caballero de quien había recibido esta misión y a quien no quiere devolver el dinero recibido por el crimen abortado. Aún peor, perseguido por la gente del comendador después de este crimen, mata también a este último antes de echarse al mar para escapar de los "ministros de la justicia".

Por fin, y será el último marco urbano que evocaremos en esta primera parte, la tercera jornada nos muestra a Enrico encarcelado. La cárcel sintetiza pues, en cierta medida por lo menos (pero no en todas, lo veremos después) todos los aspectos relacionados con el crimen (en sentido global) y la ciudad. Además de esto, la cárcel

5. Tirso de Molina, Obras dramáticas completas, t. III, p. 465.

6. En realidad, existe otro lugar relacionado con la violencia de los bandidos, y es, precisamente, el monte. Volveremos sobre este punto más adelante. 
es también el escenario del último asesinato sangriento y despiadado de Enrico que, loco de ira, mata a un portero con la cadena que lo inmovilizaba después de romperla.

Después de este rápido panorama de los lugares urbanos consagrados al crimen y antes de pasar a nuestra segunda parte, quisiéramos detenernos unos segundos para proponer un balance parcial. Nos parece importante subrayar aquí, en efecto, que la geografía dramática que acabamos de poner de relieve en estas líneas, es un primer estrato de la importancia de la ciudad en la economía de la comedia. De hecho, hay que recordar que, en la lógica interna de la comedia, la ciudad consustancialmente criminal y hasta diabólica, si recordamos quién empuja la acción hacia ella, se opone sistemáticamente, como lo vamos a ver más claramente en el segundo movimiento, al monte eremítico dedicado a la santidad o, por lo menos, a su búsqueda.

\section{DOS MUNDOS AL REVÉS}

Como acabamos de escribirlo, otro espacio en la comedia desempeña un papel clave en la economía del drama, el del monte eremítico. Este espacio constituye en efecto el segundo elemento de una estructura textual dinámica que lo hace todo para crear un efecto de perfecta reversibilidad de los dos espacios. La temporalidad de la obra se empeña en particular en poner de manera sistemática la acción en el monte en paralelismo perfecto con la acción en la ciudad. De hecho, a partir del momento en que nos interesamos en el orden de aparición de los protagonistas en el escenario o en el texto, nos percatamos del hecho de que las escenas con Paulo y aquellas con Enrico se desarrollan exactamente en el mismo momento en dos lugares distintos. A modo de ejemplo, la crisis de Paulo, su monólogo, su diálogo con el demonio y su decisión de ir a Nápoles se desarrollan exactamente en la misma temporalidad que las escenas en la casa de Celia. Y esto se comprueba cada vez que los dos protagonistas no están juntos en el escenario o en el texto. Esquemáticamente podemos resumir la doble estructura temporal y geográfica de la acción dramática de la manera siguiente: Paulo está primero en el monte mientras Enrico está en la ciudad, luego los dos se encuentran juntos primero en la ciudad, luego en el monte y por fin, Paulo acaba su vida en el monte y Enrico en la ciudad. Este esquema puede dar la impresión de una vuelta mecánica al principio de la historia (cada uno muere donde nació como personaje dramático) pero es que en realidad falta un criterio esencial para poner esta alternancia binaria en movimiento: este criterio es la salvación. De hecho, primero en el monte Paulo es el personaje salvado y en la ciudad Enrico es el condenado. Su encuentro con Enrico (Paulo presencia la escena del asesinato del mendigo y el concurso de crímenes y delitos) precipita a Paulo en el crimen; de vuelta al monte, éste se convierte en el condenado, mientras que Enrico emprende su camino hacia la salvación que se concretiza en la ciudad, al final de la obra. La inversión de las polaridades que acabamos de describir rápidamente se hace a favor de la ciudad. Dos lugares en la Nápoles dramática del Condenado participan, en particular, de este movimiento; sin embargo, antes de analizarlos nos parece necesario explicitar, aunque brevemente, en qué medida el 
monte pierde, por lo menos en esta obra en particular, su relación íntima con la santidad, su vínculo multisecular con la salvación. De hecho, bien se conoce, como lo recuerda en particular M. E. Kaufmant en su trabajo Poetique des espaces naturels, que le monte es el espacio de la purgación de las pasiones y de los pecados, el desierto de los anacoretas.

Le monte des pièces hagiographiques est bien, en premier lieu, l'espace de la solitude érémitique. Comme axis mundi, le monte est l'objet d'une poétique de l'élévation, de la verticalité. La plupart des comedias hagiographiques représentent, à un moment ou à un autre, cet espace typologique de l'ermite. Citons Paulo dans le premier acte de El condenado por desconfiado ; Eurosia et son attirance pour l'état d'ermite dans le monte de La joya de la montaña, ou encore Saint Eustache [...] dans La mayor dicha en el monte?

El monte es también el lugar privilegiado de cierta forma de violencia que es la de los bandoleros, otra especificidad tradicional del monte y vuelvo a citar a MarieEugénie Kaufmant:

[...] la principale violence représentée dans le monte est celle du bandit de grands chemins qui y trouve un retranchement idéal pour échapper à la justice. Ce n'est pas seulement parce qu'il se situe en marge de la justice que le monte est l'espace du bandit retranché dans sa topographie inaccessible. II y a un lien évident entre le monte-refuge et l'imaginaire du banditisme dans la comedia ${ }^{8}$.

En El condenado, si Paulo parece primero vivir en perfecta sintonía con el espacio de soledad eremítica en el que está, el profundo trauma que sufre después de ver su destino liado con el del espadachín asesino Enrico, lo precipita hacia la violencia bandolera de este mismo espacio:

En el monte hay bandoleros;

bandolero quiero ser,

porque así igualar pretendo

mi vida con la de Enrico,

pues un mismo fin tenemos ${ }^{9}$.

Sin embargo, esta elección codificada que hace penetrar la violencia en la topografía normalmente dedicada a la santidad (y que bien hubiera podido desembocar, de manera tópica, en una toma de conciencia y una redención de Paulo) es en realidad una perversión mucho más profunda. En efecto, se produce también al revés de lo que suele ocurrir en las obras hagiográficas en las que la huida al monte y la violencia sistemática suelen ser la consecuencia de una violencia social inicial, padecida por el protagonista que, además, suele ser una mujer (basta con pensar en la duquesa Ninfa en La Ninfa del cielo, traicionada por un hombre y que decide vengarse de todos los hombres convirtiéndose en bandolera). En esta obra,

9. Tirso de Molina, Obras dramáticas completas, t. III, p. 470. 
Paulo no se revela contra una injusticia social sino contra la de Dios, razón por la cual será condenado por el "Juez Supremo". Este paréntesis hacia un elemento a priori exterior a nuestro tema nos parece sin embargo pertinente en la medida en que la pérdida por parte del monte de la potencialidad salvadora -la intervención de Cristo, tres veces, bajo la forma de un pastor ni siquiera abrirá los ojos de Paulo, para quien el monte perdió todo su carácter sagrado- no significa que esta potencialidad haya desaparecido del todo del universo dramático de la obra, sino que se ha desplazado hacia la ciudad.

Como lo decíamos antes de esta escapada hacia el monte, dos lugares en la ciudad de Nápoles desempeñan un papel clave en la reorientación de los espacios en la comedia. Estos dos lugares son, primero la habitación de Anareto, el viejo padre de Enrico, y por otra el calabazo en que se encuentra finalmente aislado Enrico después de matar al portero de la cárcel. El nombre del viejo padre del rufián es pronunciado varias veces en la obra antes que este aparezca por fin. Casi todos hablan de él: el Demonio cuando se dirige a Paulo y le explica que su destino está enteramente relacionado con el de Enrico, hijo de Anareto; el mismo Paulo cuando le relata la aparición a su criado Pedrisco; uno de los dos marqueses, asombrado que semejante padre tenga a semejante hijo y el mismo Enrico para declarar su amor y su devoción por su padre. Todos para subrayar lo mismo: la vejez y la virtud del anciano. O sea que incluso en los corazones más pecaminosos sigue latiendo un nombre capaz de evocar para todos la pureza. Y este nombre es el de un hombre que vive en la ciudad. Anareto vive en efecto en una casa de Nápoles de la que nunca sale por estar enfermo y ser viejo y a donde viene cotidianamente Enrico para traerle de comer a su padre y cuidar de él casi como una hija. Cuando aparece por fin en el escenario, al principio de la segunda jornada, todo en la puesta en escena de esta aparición por parte del texto dramático está hecho para crear un efecto de empatía muy fuerte y también de admiración. En la misma habitación, una cortina esconde primero al protagonista y cuando la aparta Enrico descubrimos al anciano durmiendo:

[Enrico] descorre las cortinas de la alcoba y se ve a Anareto dormido en una silla.

Aquí está: quiérole ver

Durmiendo está al parecer

Padre ${ }^{10}$

Si nos detenemos en la dramatización de la vida del padre de Enrico, estamos frente a un hombre humilde, virtuoso y solitario que vive recluido en su habitación, aislado en el mismo corazón de la ciudad, habitación de la que nunca sale, que solo ve a su hijo que le trae con que alimentarse, como lo haría un penitente para con un ermitaño. Dicho de otro modo, más directo, si hay un anacoreta en esta obra, se trata claramente de Anareto. La habitación de Anareto, y más aún su alcoba,

10. Tirso de Molina, Obras dramáticas completas, t. III, p. 472. 
son la vertiente ciudadana y virtuosa de la cueva tradicional ${ }^{11}$ del ermitaño en su soledad eremítica en el monte ${ }^{12}$. Y precisamente, esta alcoba no aparece en la obra en cualquier momento. Estamos poco después del principio de la segunda jornada o sea más o menos en el mismo punto textual que cuando Paulo, al principio de la primera jornada, se encierra en su cueva para rezar. Si Anareto tiene la felicidad de recibir el amor filial de Enrico, Paulo en cambio se duerme y es víctima de una pesadilla mandada por el Demonio a quien encuentra al salir de su cueva. Este foco de santidad en medio de un universo urbano innegablemente marcado por el vicio debe ser tomado muy en serio dado que puede percibirse no tanto como un elemento virtuoso aislado en un conjunto asociado a la condenación sino como el símbolo de la estructura binariamente reversible que elabora, por lo menos en lo que se refiere a su superficialidad, la obra. Paulo, el santo ermitaño, recluido en el espacio tópico de la salvación, el monte, será condenado por su desconfianza mientras que Enrico, rufián despiadado dueño de un espacio urbano consagrado a la violencia, será salvado por su capacidad sin límites a amar a su padre cuya figura sacralizada por el texto dramático se convierte claramente en una imagen de Dios.

El segundo lugar napolitano que se convierte en la obra en un lugar transitorio hacia la salvación es el lugar donde se suele concentrar la quintaescencia del vicio: la cárcel de la ciudad. La tercera jornada empieza con Enrico encarcelado y airado. Después de matar a un portero en un último desencadenamiento de furia (su examante, Celia, ya casada con uno de los dos marqueses del principio se ha negado a ayudarle), el rufián, se encuentra encerrado solo en un calabozo. En este momento una voz misteriosa y -lo dice el mismo Enrico- aterradora, se oye ${ }^{13}$. Las acotaciones nos aprenden que se trata del Demonio, invisible. Ofrece la libertad a Enrico y le abre una puerta en la pared del calabozo. Sin embargo, también se oye otra voz que le canta al preso un consejo muy diferente:

Detente, engañado Enrico,

No huyas de la prisión;

Pues morirás si salieres,

Y si te estuvieres, no ${ }^{14}$.

11. Al principio de la obra, Paulo se refugia en su cueva (la cueva eremítica por excelencia) para meditar, pero solo consigue dormirse y tener una pesadilla que acaba de precipitarle hacia la trampa del demonio. 12. Para la importancia semiótica del espacio escénico ver Ruano de la Haza, 1997 y Rubiera, 2005. 13. Tirso de Molina, Obras dramáticas completas, t. III, p. 492. Se podría establecer un paralelismo interesante entre el diálogo de Enrico con la Voz, que aparece bajo la forma de una sombra y el diálogo entre don Juan y la estatua del Comendador sobre la cuestión del miedo: «ENRICO: ¿Cómo te puedo creer / voz, si no llego a saber / quién eres y dónde estás? DEMONIO: Pues agora me veras (Aparécesele como en forma de una sombra.) ENRICO: Ya no te quisiera ver. DEMONIO: No temas, ENRICO: un sudor frío / por mis venas se derrama. [...] / Poco de mis fuerzas fío. / No te acerques». Se ve muy bien que aquí, y contrariamente a la valentía blasfematoria de don Juan, Enrico experimenta el miedo tal como lo rezan los salmos y proverbios como, por ejemplo, el Salmo 111:10: «El principio de la sabiduría es el temor de Jehová; / Buen entendimiento tienen todos los que practican sus mandamientos; / Su loor permanece para siempre» (Salmos 111:10, Reina-Valera 1960 (RVR1960)).

14. Tirso de Molina, Obras dramáticas completas, t. III, p. 493. 
Claro, los lectores-espectadores ya han entendido que, al rechazar la huida y a pesar de sus últimas resistencias, el personaje de Enrico acaba de conseguir la vía de la salvación. Lo importante para nosotros aquí es que esta escena sobrenatural de redención - que completará la llegada de Anareto a la cárcel y su perdón- tiene, otra vez, su desdoblamiento en el monte con Paulo. Mientras, en la ciudad, la intervención divina salva a un Enrico que se abandona a la confianza del corazón frente a su padre, el pequeño pastor, desesperado y herido ("Yo tengo las plantas/de jaras diversas/y agudos espinos/rotas y sangrientas") no consigue obtener lo mismo de Paulo cuyo cerebro se sustituye al corazón: ya no siente, solo piensa, no confía, argumenta y se condena:

$$
\begin{aligned}
& \text { En lágrimas tiernas } \\
& \text { Baña el pastorcillo } \\
& \text { Las mejillas bellas. } \\
& \text { Pues te desconoce } \\
& \text { Olvídate de ella, } \\
& \text { Y no llores más }
\end{aligned}
$$

La fuerza de la fe, sencilla y entera, se instala en la ciudad mientras que le monte pierde su relación multisecular y privilegiada con el misterio y lo divino. Las ovejas perdidas ya no son parábolas sino animales.

Antes de pasar a nuestra tercera y última parte conclusiva, nos parece interesante recordar el destino de los demás personajes del drama, casi todos habitantes de la urbe: Celia, el marqués fascinado por su (¿mala?) fama, y Galván, de quien no hemos hablado hasta ahora y que es el criado de Enrico. De hecho, todos conocen un final bastante feliz. Celia renuncia a su vida libre pero inmoral casándose con el Marqués que, a su vez, abandona la frivolidad de un galán acostumbrado a amores costosos, y Galván, aunque cómplice de los crímenes de su amo, recibe el perdón del juez que estima que ha tenido bastante escarmiento con lo que acaba de vivir. Es de recordar que cuando Pedrisco, criado de Paulo, pronuncia las últimas palabras de la obra, se produce un cambio de título, que aclara mucho más que el mero destino de Paulo y de Enrico:

Y con aquesto da fin

El mayor desconfiado,

$Y$ pena y gloria trocadas.

El cielo os guarde mil años ${ }^{16}$.

El sintagma «pena y gloria trocadas» explicita en efecto una de las lecciones de la obra: cada protagonista importante se ha visto en situación de elegir entre el mal y el bien (no olvidemos que uno de los temas de la comedia es el del libre albedrío) y, cosa nueva, todos los que se han apartado del camino del pecado lo han hecho en la ciudad o sea en el mismo crisol del pecado. Habría mucho que decir, por ejemplo, sobre Celia, personaje demasiado despreciado y subestimado. Esta inversión aca- 
ba confirmándose con el final de la obra. En efecto, al final de la historia, el monte es el lugar de la condenación de Paulo (a quien vemos literalmente arder en las llamas del infierno) mientras que la ciudad es el marco de la regeneración, de la salvación de Enrico que muere ejecutado por la justicia de los hombres frente a la cual paga por sus crímenes, pero salvado por la misericordia divina.

\section{¿LA CIUDAD DE LOS PRODIGIOS?}

Para acabar con este análisis parcial del estatuto de la ciudad en El condenado por desconfiado, quisiéramos proponer unos elementos de reflexión más globales. Esperamos haber demostrado la inversión que se produce a lo largo de la obra en lo que se refiere al valor simbólico del monte y de la ciudad en una lógica de reversibilidad conforme con el programa global de la comedia, activado por sus últimos versos Pena y gloria trocadas. Sin embargo, quisiéramos matizar, para acabar, la impresión fuerte de polaridad y de dualidad que se desprende tanto de la obra como de nuestro análisis. En efecto, sería un error afirmar que la ciudad ha pasado a ser una especie de "nuevo monte", o sea, una actualización urbana de las características tradicionales del monte tanto en lo que se refiere a la purgación eremítica como a la violencia bandolera. De hecho, la ciudad nos parece emerger más bien como espacio híbrido (lo que no es necesariamente el monte, si aceptamos la idea de que dualidad, la reversibilidad de su simbolismo no es hibridez dado que participa también de la precepción de este espacio como espacio codificado de la conversión y de la redención). Esta hibridez es precisamente lo que hace, según pensamos, que la ciudad cobra los rasgos modernos que hemos evocado en la introducción de este trabajo. La obra invalida claramente la posibilidad de una salvación global mediante recursos antiguos ya caducados: los ermitaños, los anacoretas ya no pueden encarnar la solución para una fe en peligro frente al pensamiento premoderno, a una duda pre-cartesiana encarnada por Paulo cuyo mayor pecado radica in fine en su voluntad de debatir lógicamente con Dios. La elección de Nápoles como marco de esta nueva propuesta es reveladora. Por su geografía, Nápoles está precisamente entre el monte, cuna tradicional de la santidad (como lo demuestra, entre otras cosas, Ramón Menéndez Pidal en su discurso), y el mar, lugar temible que el pesimismo barroco considera como lugar peligroso y hostil. Stephen Gilman, en su obra de 1951 Cervantes y Avellanada, estudio de una imitación, analiza perfectamente bien esta percepción del mar ${ }^{17}$ que se opone en su violencia y su carácter incomprensible, caótico ${ }^{18} \mathrm{con}$ la idea de un mundo amable y que puede ser comprendido mediante los sentidos, herramienta de predilección del conocimiento humano en el pensamiento renacentista. De la misma manera, Nápoles está marcada tanto por la mitología pagana y peligrosa (habría sido fundada por la sirena Parténope) como por el cristianismo, a través de su santa patrona, Santa Lucía. Nápoles es un lugar múltiple, complejo, híbrido de por sí en la medida de que en ella se funden todas estas herencias contradictorias. Enrica Cancelliere 
recuerda en su estudio de la ciudad de Nápoles en las dos obras maestras El Burlador de Sevilla y El condenado por desconfiado que

La Porta del Mare es [... $]^{19}$ asimilada a un perno mágico del tiempo y del espacio, el umbral que puede dar lugar a sus opuestos, como la eternidad o la utopía.

A estos elementos de reflexión, tenemos que añadir otro, más complejo aún. El protagonista principal de la obra, el "condenado por desconfiado", es el ermitaño Paulo. Lleva el mismo nombre que el apóstol de los gentiles, Paulo/Pablo de Tarso. Este, se quedó tres días ciego y por fin le iluminó la fe en Cristo, mientras que la ceguera de Paulo, en su monte, se la provoca el demonio disfrazado de ángel, haciéndole emprender el camino de Damas al revés, de la fe más perfecta al más encarnado farisaísmo, en un recorrido de tres jornadas. El Paulo bíblico iba de ciudad a ciudad a evangelizar a los gentiles con toda la fuerza de su inteligencia y reclamando la sencillez del corazón. El Paulo de la obra vive en el monte, pero, y este detalle es fundamental, viene de la ciudad, no es un hijo de la tierra, sino un hijo de la urbe. A pesar de tener todas las cualidades de un caballero y de un santo no sabe ver en la ciudad, en que nació y creció, la potencialidad evangélica que late en ella y que hubiera podido salvarle (con tal que renunciara a seguir su inteligencia a favor de su corazón). O sea que, quizás, lo que propone aquí Tirso -que suele aparecer, en algunas obras, tras un personaje de pastor llamado Tarso, detalle que puede tener su importancia en la perspectiva de una lectura literal- sea la toma en cuenta de una nueva geografía de la fe que se aleja de la sencillez y de la ingenuidad alegórica de espacios poéticos eremíticos, para aceptar la ineluctabilidad de la modernidad urbana, de su confusión, pero también de su potencial riqueza.

Nuestras últimas palabras serán quizás más bien un juicio que un auténtico análisis, pero nos parece, a pesar de todo lo que acabamos de escribir, que el optimismo final de la salvación de Enrico es un poco forzado y que lo que domina en esta obra es la inquietud frente a la desaparición de un mundo ya caducado (el monte eremítico $)^{20}$ e incapaz de enfrentar los nuevos pecados de hombres que quieren comprender en vez de creer. De hecho, la presencia redentora de Anareto en el mismo corazón de la urbe debe ser matizada. Anareto es un anciano cuya debilidad e impotencia hacen imposible que veamos en él una verdadera regeneración del tema del ermitaño. Aunque salva a su hijo, y aunque éste se salva en el corazón de la ciudad de la que había intentado huir es de notar que El condenado es la única obra con El burlador en la que el protagonista acaba condenado (las dos tienen a Nápoles como escenario común). La ciudad se ha convertido en la obra en un sitio lleno de potencialidades, incluida la de la santidad, pero, el optimismo tirsiano, que suele caracterizar sus obras hagiográficas, no consigue borrar totalmente, según nosotros, la impresión de que la condena de Paulo, por muy merecida que resulte en la economía del drama en cuestión, es también un fracaso divino frente a hombres nuevos, peligrosamente argumentadores y urbanos.

19. Cancelliere, 2017, p. 98.

20. No olvidemos, en efecto, que el pequeño pastor que le aparece tres veces a Paulo, a lo largo de la obra, no consigue recuperar su oveja perdida y acaba con los pies ensangrentados. 


\section{BiBLIOgRAFÍA}

Cancelliere, Enrica, «La ciudad de Nápoles en dos comedias de Tirso de Molina y el poder del teatro», en Autorité et pouvoir dans le théâtre du siècle d'Or / Autoridad y poder en el teatro del Siglo de Oro, Bulletin Hispanique, 119, junio de 2017, pp. 89-100.

Cervantes, Miguel de, Don Quijote de la Mancha, ed. Martín de Riquer, Madrid, Planeta, 1980.

Gilman, Stephen, «Cervantes y Avellanada: estudio de una imitación», Nueva Revista de Filología hispánica, México, El Colegio de México, 1951.

Guevara, Antonio de, Menosprecio de corte y alabanza de aldea, ed. Matías Martínez Burgos, Madrid, Espasa-Calpe, 1942.

Kaufmant, Marie-Eugénie, Poétique des espaces naturels dans la comedia nueva, Madrid, Bibliothèque de la Casa Velázquez, 2010.

Menéndez Pidal, Ramón, Discurso leído ante la Real Academia acerca de «El condenado por desconfiado», 1920, disponible en <www.rae.es/sites/default/files/ discurso_ingreso_Ramon_Menendez_Pidal>.

Tirso de Molina, El burlador de Sevilla, William Hunter, Pamplona, Instituto de Estudios Tirsianos, 2008.

Tirso de Molina, Obras dramáticas completas, t. III, ed. Blanca de los Ríos, Madrid, Aguilar, 1989.

Ruano de la Haza, José María, «Una posible puesta en escena de El condenado por desconfiado», en La década de oro en la comedia española: 1630-1640, ed. Felipe B. Pedraza y Rafael González Cañal, Almagro, Universidad de CastillaLa Mancha / Festival de Almagro, 1997, pp. 103-126.

Rubiera, Javier, La construcción del espacio de la comedia española del Siglo de Oro, Madrid, Arco/Libros, 2005. 